\title{
Pain Management in Enhanced Recovery after Surgery (ERAS) Protocols
}

\author{
J. Creswell Simpson, MD ${ }^{1}$ Xiaodong Bao, MD, $\mathrm{PhD}^{1} \quad$ Aalok Agarwala, MD, MBA ${ }^{1}$ \\ ${ }^{1}$ Department of Anesthesia, Critical Care and Pain Medicine, \\ Massachusetts General Hospital, Boston, Massachusetts \\ Address for correspondence Aalok Agarwala, MD, MBA, Division of \\ General Surgery Anesthesia, Department of Anesthesia, Critical Care \\ and Pain Medicine, Massachusetts General Hospital, 55 Fruit Street, \\ Clin Colon Rectal Surg 2019;32:121-128. \\ GRB 444, Boston, MA 02114 (e-mail: aagarwala@mgh.harvard.edu).
}

\begin{abstract}
Keywords

- ERAS

- multimodal analgesia

- pain management

- colorectal surgery

Pain control is an integral part of Enhanced Recovery after Surgery (ERAS) protocols for colorectal surgery. While opioid therapy remains the mainstay of therapy for postsurgical pain, opioids have undesired side effects including delayed recovery of bowel function, respiratory depression, and postoperative nausea and vomiting. A variety of nonopioid systemic medical therapies as well as regional and neuraxial techniques have been described as improving pain control while reducing opioid use. Multimodal and preemptive analgesia as part of an ERAS protocol facilitates early mobility and early return of bowel function and decreases postoperative morbidity. In this review, we examine several multimodal therapies and their impact on postoperative analgesia, opioid use, and recovery for patients undergoing colorectal surgery.
\end{abstract}

Enhanced Recovery after Surgery (ERAS) protocols are rapidly becoming the standard of care for patients undergoing elective colorectal surgery to speed recovery and decrease postoperative length of stay (LOS) and morbidity. While these protocols encompass numerous interventions including modified preoperative oral intake, avoidance of mechanical bowel preparation, early postoperative mobilization and oral intake, and emphasis on laparoscopic surgical technique, one of the cornerstones of ERAS protocols is the use of multimodal analgesic therapy. Multimodal analgesia includes a variety of pharmacologic, regional, and neuraxial modalities aimed at reducing opioid consumption and thus opioid-related side effects.

\section{Opioid Analgesia and Side Effects}

Opioids are an effective therapy for severe pain, and their use remains a mainstay of treatment for pain control after surgery. However, use of opioid medication comes with significant side effects including ileus, respiratory depression, and nausea and vomiting.

\section{Postoperative lleus}

Opioid therapy has been implicated in postoperative ileus and prolonged recovery of bowel function in a dose-depen- dent manner. Ileus can be an especially devastating complication in patients following colorectal surgery in whom return of bowel function is a key part of recovery. In a 2011 study of patients undergoing colorectal surgery, Barletta et al found that a total daily hydromorphone dose of greater than $2 \mathrm{mg} /$ day was significantly associated with development of ileus; this risk was almost 10 -fold higher in patients undergoing open procedures ( 2.6 vs. $22 \%)^{1}$ Though absolute rates were lower, risk of ileus was also significantly increased for patients undergoing laparoscopic colorectal surgery. In addition to increased rates of ileus, patients with a total daily hydromorphone dose exceeding $2 \mathrm{mg}$ per day had a mean LOS that was 2 days longer for open cases and 1 day longer for laparoscopic cases.

Studies examining opioid-sparing analgesic regimens have shown a decrease in postoperative ileus. Lidocaine, ${ }^{2}$ celecoxib, ${ }^{3}$ magnesium, ${ }^{4}$ and epidural analgesia ${ }^{5}$ have all been shown to decrease opioid consumption and decrease risk of postoperative ileus formation. Postoperative ileus contributes significantly to both cost and LOS in patients undergoing colorectal surgery. An analysis by Asgeirsson et al showed that hospital costs were doubled for patients who developed postoperative ileus, ${ }^{6}$ and a retrospective study by Vather and Bissett showed an increase in average LOS from 6 to 13 days. $^{7}$
Issue Theme Enhanced Recovery after Surgery (ERAS) for Colorectal Surgery; Guest Editor: Hiroko Kunitake, MD, MPH
Copyright $\odot 2019$ by Thieme Medical Publishers, Inc., 333 Seventh Avenue, New York, NY 10001, USA. Tel: +1(212) 584-4662.
DOI https://doi.org/ 10.1055/s-0038-1676477. ISSN 1531-0043. 


\section{Postoperative Respiratory Depression}

One of the most feared complications of narcotic use is opioid-induced respiratory depression. While it is rare compared with postoperative ileus, opioid-induced respiratory depression can have devastating consequences. An analysis of the Anesthesia Closed Claims Project database from 1990 to 2009 by Lee et al found that 92 out of the 9,799 claims in the database had "possible, probable, or definite" opioidinduced respiratory depression events. Of these 92 claims, $77 \%$ resulted in severe brain damage or death; the vast majority of these events (88\%) occurred within 24 hours of surgery. ${ }^{8}$

\section{Postoperative Nausea and Vomiting}

Postoperative opioid use is a significant risk factor for postoperative nausea and vomiting (PONV). A 2012 meta-analysis by Apfel et al identified opioid use as one of the four major anesthesia-related factors contributing to PONV. ${ }^{9}$ PONV contributes to prolonged LOS in the postanesthesia care unit (PACU); in addition, patients often rate PONV as worse than postoperative pain. Vomiting has been associated with aspiration, wound dehiscence, esophageal rupture, and pneumothorax. $^{10}$

Use of nonopioid analgesics has been shown to decrease PONV in patients undergoing colorectal surgery. Lidocaine, ${ }^{11}$ gabapentin, ${ }^{12}$ ketamine, ${ }^{13}$ pregabalin, ${ }^{14}$ acetaminophen, ${ }^{15}$ as well as regional ${ }^{16}$ and neuraxial ${ }^{5}$ techniques have all been shown to decrease the incidence of PONV in colorectal surgery patients compared with opioid-based analgesia alone.

\section{Multimodal Analgesia}

Multimodal analgesia has been defined as "use of more than one modality of pain control to achieve effective analgesia while reducing opioid-related side effects." ${ }^{17}$ Given the complications associated with the use of opioid therapy for postoperative pain, multimodal techniques have emerged as a way to allow for similar-or in some cases improved-pain control while minimizing opioid use. Multimodal analgesia encompasses both systemic medication administration as well as regional and neuraxial techniques. Multimodal analgesia also incorporates the idea of preemptive analgesia, or the administration of medication to reduce pain before surgery or painful stimulus has occurred. Preemptive, rather than reactive, use of analgesic medication has been shown to reduce pain, inflammation, and PONV. ${ }^{15,18}$ The ERAS Group published consensus recommendations for colorectal surgery which highlight nonopioid analgesia, both systemic and neuraxial, as a key part of an ERAS protocol based on benefits with respect to postoperative return of bowel function, pain control, and morbidity. ${ }^{19}$

\section{Systemic Therapy}

\section{Acetaminophen}

Acetaminophen is a mainstay of adjuvant analgesic therapy. Acetaminophen has a favorable safety profile and has been shown to decrease postoperative opioid consumption. A 2010 review by Maund et al examining 12 trials of postoperative acetaminophen versus placebo showed a decrease in morphine consumption of $8.68 \mathrm{mg}$ over the first 24 hours, though they did not find any improvement in PONV or sedation. ${ }^{20}$ Acetaminophen is particularly effective in synergy with nonsteroidal anti-inflammatory drugs (NSAIDs). A 2010 systematic review of 21 studies including over 1,900 patients undergoing a variety of surgical procedures demonstrated a mean reduction in visual analog scale (VAS) for pain scores of $35 \%$ and a reduction in opioid analgesic dose of $38.8 \%$ with a combination of acetaminophen and NSAIDs versus acetaminophen alone. A similar benefit was seen with the combination versus NSAIDs alone, with reductions of 37.7 and $31.3 \%$, respectively. ${ }^{21}$

Acetaminophen is available in both oral and intravenous (IV) preparations, though the latter is considerably more expensive. IV acetaminophen has become a popular adjunct in place of oral or rectal acetaminophen given the ease of administration, particularly in patients who cannot take oral medications. IV acetaminophen has been shown to have both a faster time to peak cerebrospinal fluid (CSF) levels as well as a much larger CSF concentration-area under the curve (AUC); however, direct comparison studies between IV and oral acetaminophen have failed to show a benefit in terms of pain control or opiate requirement in patients undergoing total joint arthroplasty ${ }^{22}$ or oral surgery. ${ }^{23}$ Direct comparison studies of IV acetaminophen versus oral or rectal routes for abdominal surgery are lacking. Absorption of rectal acetaminophen has been shown to be quite variable (though in a very small population) with peak CSF levels and AUC less than half of those in the IV group and 50\% less than the PO group. ${ }^{24}$

A 2013 meta-analysis by Apfel et al showed a decrease in PONV with use of IV acetaminophen. In a review of 30 trials encompassing 2,300 patients, they found a relative risk (RR) of 0.73 for nausea and 0.63 for vomiting leading to a number needed to treat (NNT) of 12 and 14 , respectively. ${ }^{15}$ Decreased rates of PONV from the study of Apfel et al were maintained whether acetaminophen was given intraoperatively, preoperatively, or postoperatively as a prophylactic measure; however, it was not effective if given as a reactive measure after patients experienced postoperative pain.

\section{Lidocaine}

Lidocaine is an amide local anesthetic and antiarrhythmic agent that blocks voltage-gated sodium channels in neurons, preventing depolarization and inhibiting the propagation of nerve impulses. Lidocaine infusions have been shown to be a safe and effective adjunct for multimodal pain control. A Cochrane review from 2015 examining 45 trials with 2,502 subjects found that lidocaine infusions significantly reduced postoperative pain in the first 24 hours postoperatively. ${ }^{2}$ This effect was most pronounced for laparoscopic abdominal surgery. In addition, their review found that risk of postoperative ileus was significantly reduced by IV lidocaine with an RR of 0.38. Lidocaine also reduced overall hospital LOS, opioid requirements, and PONV. 
Lidocaine may also have a beneficial effect on inflammatory mediators. A three-armed study examining thoracic epidural analgesia, lidocaine, and placebo for patients undergoing colon surgery not only found a significant decrease in opioid requirement and faster return of bowel function but also showed significantly lower levels of inflammatory cytokines interleukin 6 and interleukin 8 in patients who were given IV lidocaine versus controls. ${ }^{25}$

Studies have not been able to demonstrate efficacy of lidocaine infusions for pain beyond 24 hours. A meta-analysis by Vigneault et al in 2011 showed improved pain scores and decreased morphine requirement at 6, 12, and 24 hours but failed to find any benefit at later time points. ${ }^{11}$ Grady et al in 2015 failed to find any benefit to lidocaine in addition to ketamine or either drug alone versus placebo in terms of effect on 6-minute walk distance on postoperative day (POD) 2 in abdominal hysterectomy patients. ${ }^{26}$

Optimal dosing of IV lidocaine is not clear. The standard dosing regimen in the trials reviewed by the Cochrane group was $2 \mathrm{mg} / \mathrm{kg} /$ hour; however, significant heterogeneity existed as far as duration of infusion, which ranged from intraoperative use only up to 48 hours postoperatively. ${ }^{2}$ Despite lack of clear data regarding optimal dosing regimen or timing, neither of the two large meta-analyses referenced previously were able to find any significant increase in adverse events as a result of perioperative IV lidocaine use.

\section{Gabapentinoids}

Gabapentin and pregabalin (collectively referred to as "gabapentinoids") are antiepileptic drugs which have traditionally been used as therapies for chronic and neuropathic pain; however, their use in the acute pain arena has increased recently as data have shown benefit for perioperative pain control and nausea. While gabapentinoids have structural similarity to the inhibitory neurotransmitter $\gamma$-aminobutyric acid (GABA) receptor, their mechanism of action is not well understood. A randomized controlled trial (RCT) of 60 patients undergoing abdominal hysterectomy demonstrated a substantial decrease in postoperative tramadol use in patients who received a single 1,200 $\mathrm{mg}$ dose of gabapentin preoperatively. Tramadol use was $420 \mathrm{mg}$ in the control group versus $270 \mathrm{mg}$ in the intervention group. ${ }^{27}$ Sen et al examined gabapentin or ketamine versus placebo in hysterectomy patients and showed that patients who received gabapentin $1,200 \mathrm{mg}$ orally preoperatively required $42 \%$ less opioid medication over the first 24 hours postoperatively, which was similar to the group which received intraoperative ketamine (35\% reduction). Patients who received gabapentin (but not patients who received ketamine) also had a decrease in self-reported pain scores in follow-up phone interviews at 1,3 , and 6 months postoperatively, suggesting gabapentin may have a role in reducing development of chronic pain after surgery. ${ }^{28}$

In addition, gabapentin may have a benefit on PONV. A recent meta-analysis of 1,605 patients showed a reduction in PONV for patients who received preoperative gabapentin. The RR was 0.76 for postoperative nausea, 0.63 for postoperative vomiting, and 0.6 for use of rescue antiemetic medication for patients who received gabapentin versus controls. $^{12}$

Pregabalin has also been used as an adjunct for multimodal analgesic therapy. An RCT examining pregabalin 150 mg every 8 hours starting preoperatively and continuing for 5 days postoperatively showed significantly lower morphine consumption at $4,8,24$, and 48 hours in the intervention group. No increase in sedation was noted; however, there was an increase in dizziness, diplopia, and ataxia in patients receiving pregabalin. ${ }^{14}$

While individual studies have not shown an increase in side effects in patients receiving gabapentin, a recent retrospective review by Cavalcante et al showed a significant increase in respiratory depression in these patients. ${ }^{29}$ They retrospectively reviewed 8,500 patients who underwent laparoscopic surgery and found that gabapentin use was associated with an odds ratio (OR) of 1.47 for respiratory depression during phase I recovery in the PACU. Patients who experienced respiratory depression had a longer phase I recovery and an increased rate of admission to higher level of care. These risks were increased for those receiving intrathecal opioids, large doses of IV opioids, and older patients. Increasing age in particular was correlated with increased risk, with a univariate analysis showing an OR of 1.43 for age between 60 and 69 years and OR of 1.86 for patients older than 70 years. ${ }^{29}$

\section{Nonsteroidal Anti-inflammatory Drugs}

The term NSAID describes a group of nonsteroidal drugs that have anti-inflammatory, antipyretic, and analgesic effects. Most NSAIDs act via inhibition of cyclooxygenase-1 (COX-1) and cyclooxygenase-2 (COX-2), enzymes which play a role in the downstream signaling of both inflammation and pain. NSAIDs are a mainstay of multimodal analgesic therapy and have been shown to decrease postoperative opioid requirements as well as PONV. ${ }^{20}$ NSAIDs have also shown synergy with acetaminophen in terms of postoperative pain relief. One review encompassing 21 studies with patients undergoing a variety of surgical procedures showed a reduction in postoperative VAS scores of 35\% and opioid dose of $38.8 \%$ for patients treated with a combination of NSAIDs and acetaminophen. ${ }^{21}$ A 2004 RCT comparing patient-controlled analgesia (PCA) with morphine plus ketorolac to morphine PCA alone for patients undergoing open colorectal surgery showed a significant decrease in morphine consumption over the first 24 hours (66 vs. $80 \mathrm{mg}$ ) and a decrease to return of bowel function of 0.6 days. ${ }^{30}$

COX-2 selective inhibitors (coxibs) have also been proposed as an analgesic adjunct to achieve analgesia while reducing side effects associated with nonselective drugs. An RCT of patients undergoing laparoscopic cholecystectomy showed that coxibs are effective at reducing both pain and opioid requirements in this population ${ }^{31}$; in addition, patients in this study who received NSAID therapy had lower rates of sedation, itchiness, urinary retention, and fatigue. However, another study examining diclofenac or celecoxib versus placebo in patients undergoing open colorectal surgery failed to show improvement in pain control or total 
narcotic used with either NSAID, although patients receiving celecoxib had a 10 -fold reduction in rates of postoperative ileus ( 1 vs. $10 \%, p<0.05) .^{32}$

While NSAIDs have shown excellent analgesic effectiveness, studies have raised concerns about an increased risk of anastomotic leak associated with NSAID use. One retrospective study showed that use of nonselective NSAIDs was associated with a higher rate of anastomotic leak as compared with controls (14.5 vs. 9.9\% overall, OR: 2.13 ). Longer duration of NSAID use correlated with higher risk of leak. However, no increased risk of anastomotic leak was seen in patients taking COX-2 selective inhibitors. ${ }^{33}$

\section{NMDA Receptor Antagonists}

Ketamine, a N-methyl-D-aspartate (NMDA) receptor antagonist, is used for both pain control and general anesthesia. It's minimal effects on respiratory drive and strong analgesic effect have led to its popularity as an adjunct to opioid therapy for pain control; however, concern about psychomimetic effects in conscious patients have limited its role.

A meta-analysis by Laskowski et al in 2011 including 4,701 total patients undergoing a variety of surgical procedures showed that ketamine decreased postoperative opioid requirements, increased time to first rescue analgesic dose postoperatively, decreased VAS scores, and decreased PONV. These effects were most pronounced for patients undergoing abdominal and thoracic surgical procedures. However, after excluding trials where ketamine was not efficacious, a significant increase in neuropsychiatric side effects was noted (7.7 vs. 3\%). Their study did not reveal a relevant dosedependent effect of ketamine and significant heterogeneity existed between studies with respect to total ketamine dose and timing. ${ }^{13}$

An RCT by Zakine et al of patients undergoing major abdominal surgery compared analgesia in patients given a single dose of $0.5 \mathrm{mg} / \mathrm{kg}$ ketamine intraoperatively, $0.5 \mathrm{mg} /$ $\mathrm{kg}$ intraoperative ketamine plus $2 \mu \mathrm{g} / \mathrm{kg} / \mathrm{min}$ postoperative infusion, and controls. ${ }^{34}$ They found an almost $50 \%$ reduction in morphine requirement in the perioperative group (27 vs. $48 \mathrm{mg}$ morphine equivalent dose [MED]) versus intraoperative or control groups. VAS scores were also lower in both the intraoperative and perioperative groups at 4, 24, and 48 hours versus controls. They reported no neuropsychiatric side effects in any of their three groups. ${ }^{34}$

Ketamine may also decrease perioperative inflammation. A 2012 systematic review examined 11 studies looking at pro- and anti-inflammatory markers (interleukin-6 [IL-6] and IL-10, respectively) postoperatively in patients who did and did not receive ketamine as part of general anesthesia. They found a significant decrease in the proinflammatory IL-6 levels and a correlated increase in anti-inflammatory IL-10 levels; these effects seemed to be most pronounced with patients undergoing cardiopulmonary bypass. Their analysis did not detect a dose-dependent effect but had a high degree of heterogeneity in terms of dosing between studies. $^{35}$

While ketamine has been proposed as a possible adjunct to reduce chronic postsurgical pain, there remains disagree- ment in the literature about its effectiveness for this indication. One study did demonstrate a reduction in chronic postoperative pain in patients undergoing total hip arthroplasty $^{36}$; however, a study on patients undergoing abdominal hysterectomy showed improved pain scores immediately postoperatively but failed to find any longterm benefit to pain scores at 1, 3, or 6 months between ketamine and placebo. ${ }^{28}$

Magnesium has also been raised by some as a possible adjunct due to its antagonism at the NMDA receptor. Early reviews failed to find any evidence of benefit for magnesium administration $^{37}$; however, a small recent RCT examining magnesium versus placebo in open abdominal surgery showed a decrease in postoperative morphine consumption as well as a remarkable decrease in postoperative ileus, with average ileus duration of 2.3 days in the magnesium group versus 4.2 days in the control group. ${ }^{4}$

\section{Dexamethasone}

The corticosteroid dexamethasone has well-established effects on reduction of PONV ${ }^{10}$ but its role as an analgesic has been less well established. A meta-analysis from Waldron et al in 2013 showed that a single perioperative dose of dexamethasone reduced both VAS scores and morphine consumption over the first 24 hours after surgery in patients who had open abdominal procedures; however, the overall effects were relatively modest with a mean difference in morphine consumption of $-2.33 \mathrm{mg}$ ( $\sim 10 \%$ of total morphine consumption in the intervention group). Interestingly, the improvement in pain scores was preserved when only patients undergoing laparoscopic surgery were considered but the difference in morphine consumption was not. ${ }^{38}$ The most common dose of dexamethasone given in the studies analyzed was $8 \mathrm{mg}$, though doses ranged from 1.25 to $20 \mathrm{mg}$; no difference was observed when pooled data were compared between 4 to $5 \mathrm{mg}$ and 8 to $10 \mathrm{mg}$ doses.

Dexamethasone can have a potent immunosuppressive effect which has led to concerns about an increase in wound infection rates in patients who receive it intraoperatively. A retrospective analysis by Bolac et al on women undergoing laparotomies for endometrial cancer failed to show any difference in wound complication rates among patients who received intraoperative dexamethasone. ${ }^{39}$ This was corroborated by a 2015 RCT in patients undergoing colorectal surgery where no difference was observed between patients who received $4 \mathrm{mg}$ of dexamethasone versus placebo. ${ }^{40}$

Dexamethasone has been shown to potentially raise blood glucose levels in the first 24 hours postoperatively, though the clinical relevance of this finding is unclear as it has not been correlated to an increase in side effects. ${ }^{38}$

\section{Central $\alpha-2$ Agonists}

Central $\alpha-2$ agonists decrease postsynaptic release of norepinephrine and are thought to play a role in central pain modulation. Clonidine and, more recently, dexmedetomidine both have been explored as possible adjunctive medications for postoperative pain control. A randomized trial of patients undergoing laparoscopic bariatric surgery showed a 
decrease in rescue narcotic required in the PACU as well as a decrease in time to PACU discharge for patients who received intraoperative dexmedetomidine as compared with controls; however, they failed to show any change in 24-hour opioid requirements or other complications. ${ }^{41}$

A meta-analysis by Blaudszun et al examined the impact of both dexmedetomidine and clonidine on postoperative pain and morphine requirements. Their analysis found that both clonidine and dexmedetomidine decreased morphine requirements at 12 and 24 hours postoperatively. Interestingly, while clonidine's effect was most pronounced within the first 12 hours (mean morphine use decreased by $9.8 \mathrm{mg}$ at 12 hours vs. $4.1 \mathrm{mg}$ at 24 hours), the effect of dexmedetomidine was more pronounced over time-decreased by $6 \mathrm{mg}$ at 12 hours and by $14.5 \mathrm{mg}$ at 24 hours. Both clonidine and dexmedetomidine were shown to decrease PONV in their analysis (NNT of 8.9 and 9.3, respectively). However, cardiovascular side effects were common: patients who received dexmedetomidine had significantly higher rates of bradycardia (number needed to harm [NNH]: 3.1) and patients who received clonidine had higher rates of intraand postoperative hypotension (NNH: 9 and 20). ${ }^{42}$

\section{Esmolol}

Intraoperative $\beta$-blockade has gained interest as a therapy to improve both hemodynamic response to surgical stimuli and postoperative pain control. A 2007 RCT examined esmolol infusions versus fentanyl boluses or remifentanil infusions for patients undergoing laparoscopic cholecystectomy. Rescue fentanyl administration in the PACU was significantly decreased in patients who received intraoperative esmolol, with an average of 91 versus $168 \mu \mathrm{g}$ in the control group and $237 \mu \mathrm{g}$ in the remifentanil group. Patients who received esmolol infusions also met discharge criteria from the PACU and left the hospital faster than patients who received remifentanil. ${ }^{43}$

A 2004 RCT by Chia et al examining intraoperative esmolol in patients undergoing open hysterectomy also showed a significant improvement in opioid requirement. Patients who received intraoperative esmolol consumed $37.3 \mathrm{mg}$ of morphine over PODs 1 to 3 versus $54.7 \mathrm{mg}$ for patients in the control group. ${ }^{44}$

\section{Regional Anesthetic Techniques}

\section{Thoracic Epidural Analgesia}

Thoracic epidural analgesia has been used as an effective analgesic technique for both open and laparoscopic abdominal surgery, and it is identified as a key part of postoperative pain control in colorectal surgery in the ERAS group recommendations. ${ }^{19}$

Epidural analgesia provides excellent pain control while minimizing narcotic use and promoting return of bowel function. Block et al showed in a 2004 meta-analysis that epidural analgesia has improved postoperative VAS scores versus parenteral opioids for a variety of surgical procedures, including abdominal surgery. ${ }^{45}$ A subsequent meta-analysis examining epidural analgesia solely in patients who under- went colorectal surgery showed improved VAS scores at 24 and 48 hours as well as decreased opioid use. In addition, this analysis by Marret et al showed shortened duration of postoperative ileus for patients who received epidurals by an average of 36 hours. ${ }^{46}$ A 2016 Cochrane review confirmed improved pain control and faster return to bowel function in patients who received epidural analgesia for colorectal surgery. $^{47}$

Even excluding postoperative ileus, epidural analgesia may have advantageous effects on postoperative morbidity and mortality. A 2014 meta-analysis showed that epidural analgesia significantly reduced postoperative mortality, though this finding did not reach significance when cardiac surgical trials were excluded. In addition, rates of postoperative atrial fibrillation, supraventricular tachycardia, respiratory depression, atelectasis, pneumonia, ileus, and PONV were significantly decreased with epidural analgesia versus controls. $^{5}$

Disagreement exists on the impact of epidural analgesia on hospital LOS. Studies have shown improvement in LOS for open colorectal surgery in patients who receive epidurals ${ }^{48}$; however, other analyses have failed to find any benefit. ${ }^{46} \mathrm{~A}$ recent Cochrane meta-analysis showed decreased LOS for patients who underwent open but not laparoscopic surgery. ${ }^{47}$ Other studies have shown either no difference in $\operatorname{LOS}^{49}$ or even increased $\operatorname{LOS}^{50}$ in patients undergoing laparoscopic colorectal surgery who received epidurals.

The role of epidural analgesia in patients undergoing laparoscopic surgery is less well defined than in those undergoing open procedures. A 2013 meta-analysis by Khan et al showed improved postoperative pain control in patients who received epidurals for laparoscopic colorectal surgery; however, they found no impact on overall LOS. ${ }^{49}$ Other studies have failed to show any benefit for postoperative recovery of bowel function or complications from epidural analgesia in these patients. ${ }^{50,51}$

Serious complications from epidural analgesia such as hematoma and abscess are feared but extremely rare, with no events reported in any of the meta-analyses examining epidural versus PCA analgesia. However, less morbid complications are more common. Hypotension has been shown as a consequence of epidural analgesia in many but not all studies. $^{45,46,49}$ Both pruritus and urinary retention are known complications of epidural analgesia ${ }^{5}$; the latter results in many institutions leaving urinary catheters in place while patients are receiving epidural analgesia, which has been attributed to an increased incidence of urinary tract infections in these patients. ${ }^{51}$

\section{Intrathecal Morphine}

Intrathecal opioid administration has been suggested as a possible alternative to epidural analgesia for postoperative pain control. Intrathecal injection results in less pain during placement and requires less time than epidural placement. ${ }^{52}$ Intrathecal injection examining local anesthetic plus morphine versus local anesthetic alone showed a decrease in parenteral opioid requirements at 24 hours ( 7 vs. $25 \mathrm{mg}$ ) and 48 hours (11.5 vs. $31 \mathrm{mg}$ ) while improving pain control in 
patients undergoing laparoscopic colorectal surgery. ${ }^{53} \mathrm{~A}$ subsequent study by Wongyingsinn et al on patients undergoing laparoscopic colorectal surgery with an ERAS protocol confirmed an improvement in postoperative pain control and opioid requirement but failed to show a benefit in postoperative ileus or hospital LOS. ${ }^{54}$

An RCT of intrathecal morphine versus epidural analgesia in laparoscopic colorectal surgery showed improved postoperative pain control, early return to mobility, and shorter hospital LOS for the intrathecal morphine group. ${ }^{55}$ In addition, patients in the intrathecal morphine group had lower rates of insertion failure, hypotension, and sedation versus patients receiving epidural analgesia.

In contrast, a 2014 RCT examining intrathecal morphine versus epidural analgesia for open gastrectomy actually showed worse outcomes in the intrathecal group, suggesting that intrathecal morphine may be inadequate for postoperative pain control for open cases. ${ }^{52}$ In this study, patients who received intrathecal morphine consumed significantly more fentanyl, had higher rates of postoperative ileus, and had longer latency to ambulation than patients who received epidural analgesia.

\section{Transversus Abdominis Plane Blocks}

The transversus abdominis plane (TAP) block is an analgesic technique which delivers local anesthetic to the plane between the internal oblique and transversus abdominis muscles to anesthetize the thoracolumbar nerves delivering sensation to the anterior abdominal wall. TAP blocks were originally described based on a landmark-guided technique but have evolved to predominantly ultrasound-guided, ${ }^{56}$ though meta-analyses have failed to demonstrate superiority of ultrasound over landmark guidance. ${ }^{57}$

A 2010 Cochrane review found evidence that TAP blocks for abdominal surgery reduced morphine requirements over the first 48 hours postoperatively as well as increased the latency to request for first rescue analgesic dose; however, their analysis showed no evidence of effect on sedation or PONV. ${ }^{56}$ These data were based on several moderately sized studies with significant heterogeneity between them. A more recent meta-analysis by Brogi et al, however, found that while TAP blocks were effective for a variety of gynecologic and urologic procedures, when only the data for major abdominal surgery (excluding appendectomy) were analyzed there was no significant reduction in pain scores or morphine requirements over the first 24 hours. ${ }^{57}$ Interestingly, while their analysis had insufficient numbers to examine TAP blocks versus epidural analgesia, a subgroup analysis of TAP blocks versus intrathecal morphine in women undergoing cesarean sections showed significantly improved pain scores and morphine consumption in the intrathecal morphine group versus the TAP group. ${ }^{57}$

TAP blocks have also been examined for possible analgesic benefit in laparoscopic surgery. A 2013 RCT by Walter et al examining TAP blocks for laparoscopic colorectal surgery showed a decrease in 24-hour morphine requirement of $20 \mathrm{mg}$ ( 60 vs. $40 \mathrm{mg}, p<0.05$ ), though they did not show any significant difference in VAS scores, LOS, or morbidity in the intervention group. ${ }^{58}$ Interestingly, patients who received TAP blocks in this study actually had an increased incidence of PONV, though the mechanism behind this is unclear and it has not been seen in prior studies. ${ }^{16}$

Data for TAP blocks in laparoscopic colorectal surgery remains quite mixed, with several recent trials failing to show any benefit, ${ }^{59,60}$ while a recent RCT examining the combination of wound infiltration and TAP block versus infiltration alone showed benefit in terms of both opioid sparing and reduction in postoperative nausea and ileus. ${ }^{61}$ Further data are necessary to clarify which patient populations may benefit.

\section{Combining Multimodal Therapies}

While numerous studies have been conducted on individual drugs and regional techniques, the best "overall" multimodal analgesic plan remains unclear and adjuncts used vary widely between institutions. In an effort to reduce confounders, a majority of trials on multimodal therapy focus on a single intervention and preclude the use of other adjuncts. A 2009 study by Gilron et al found no benefit to the combination of meloxicam and gabapentin versus gabapentin alone in patients undergoing laparoscopic cholecystectomy, ${ }^{62}$ and a 2015 study by the same group examining triple versus double nonopioid therapy for open abdominal hysterectomy found no improvement with a third nonopioid adjunct. ${ }^{63}$ Polypharmacy has known dangers, especially in geriatric populations, where cohort studies have demonstrated polypharmacy as a risk factor for delirium ${ }^{64}$; also, as cited earlier, gabapentin has been implicated in respiratory depression, especially in older patients. ${ }^{29}$ Further research is necessary to clarify the benefits-and risks-of combining several multimodal techniques into a single protocol.

\section{Conclusion}

Multimodal analgesia as part of an ERAS protocol improves postoperative pain control while reducing opiate use. Multimodal therapy also reduces several postoperative complications including PONV and ileus formation. In patients having colorectal surgery, return of bowel function is a key indicator of recovery; ileus formation leads to substantially increased LOS and hospital costs. Use of multimodal therapy speeds return of bowel function and may therefore reduce hospital cost and LOS, especially as part of an overall ERAS program.

Early and effective analgesia is an integral part of ERAS, decreasing hospital LOS and postoperative complications. While the ideal multimodal regimen remains to be determined, it is clear that multimodal analgesic therapy has significant benefits over opiate therapy alone. Multimodal analgesia is an absolute necessity as part of any ERAS protocol for colorectal surgery and conveys substantial benefits.

Conflict of Interest

None declared. 


\section{References}

1 Barletta JF, Asgeirsson T, Senagore AJ. Influence of intravenous opioid dose on postoperative ileus. Ann Pharmacother 2011;45 (7-8):916-923

2 Kranke P, Jokinen J, Pace NL, et al. Continuous intravenous perioperative lidocaine infusion for postoperative pain and recovery. Cochrane Database Syst Rev 2015;7(07):CD009642

3 Raju DP, Hakendorf P, Costa M, Wattchow DA. Efficacy and safety of low-dose celecoxib in reducing post-operative paralytic ileus after major abdominal surgery. ANZ J Surg 2015;85(12): 946-950

4 Shariat Moharari R, Motalebi M, Najafi A, et al. Magnesium can decrease postoperative physiological ileus and postoperative pain in major non laparoscopic gastrointestinal surgeries: a randomized controlled trial. Anesth Pain Med 2013;4(01):e12750

5 Pöpping DM, Elia N, Van Aken HK, et al. Impact of epidural analgesia on mortality and morbidity after surgery: systematic review and meta-analysis of randomized controlled trials. Ann Surg 2014;259(06):1056-1067

6 Asgeirsson T, El-Badawi KI, Mahmood A, Barletta J, Luchtefeld M, Senagore AJ. Postoperative ileus: it costs more than you expect. J Am Coll Surg 2010;210(02):228-231

7 Vather R, Bissett IP. Risk factors for the development of prolonged post-operative ileus following elective colorectal surgery. Int J Colorectal Dis 2013;28(10):1385-1391

8 Lee LA, Caplan RA, Stephens LS, et al. Postoperative opioidinduced respiratory depression: a closed claims analysis. Anesthesiology 2015;122(03):659-665

9 Apfel CC, Heidrich FM, Jukar-Rao S, et al. Evidence-based analysis of risk factors for postoperative nausea and vomiting. $\mathrm{Br} \mathrm{J}$ Anaesth 2012;109(05):742-753

10 Apfel CC, Korttila K, Abdalla M, et al; IMPACT Investigators. A factorial trial of six interventions for the prevention of postoperative nausea and vomiting. N Engl J Med 2004;350(24): 2441-2451

11 Vigneault L, Turgeon AF, Côté D, et al. Perioperative intravenous lidocaine infusion for postoperative pain control: a meta-analysis of randomized controlled trials. Can J Anaesth 2011;58(01): 22-37

12 Achuthan S, Singh I, Varthya SB, Srinivasan A, Chakrabarti A, Hota D. Gabapentin prophylaxis for postoperative nausea and vomiting in abdominal surgeries: a quantitative analysis of evidence from randomized controlled clinical trials. Br J Anaesth 2015;114(04): 588-597

13 Laskowski K, Stirling A, McKay WP, Lim HJ. A systematic review of intravenous ketamine for postoperative analgesia. Can J Anaesth 2011;58(10):911-923

14 Fassoulaki A, Melemeni A, Tsaroucha A, Paraskeva A. Perioperative pregabalin for acute and chronic pain after abdominal hysterectomy or myomectomy: a randomised controlled trial. Eur J Anaesthesiol 2012;29(11):531-536

15 Apfel CC, Turan A, Souza K, Pergolizzi J, Hornuss C. Intravenous acetaminophen reduces postoperative nausea and vomiting: a systematic review and meta-analysis. Pain 2013;154(05): 677-689

16 Johns N, O'Neill S, Ventham NT, Barron F, Brady RR, Daniel T. Clinical effectiveness of transversus abdominis plane (TAP) block in abdominal surgery: a systematic review and meta-analysis. Colorectal Dis 2012;14(10):e635-e642

17 Tan M, Law LS, Gan TJ. Optimizing pain management to facilitate Enhanced Recovery After Surgery pathways. Can J Anaesth 2015; 62(02):203-218

18 Pandazi A, Kapota E, Matsota P, Paraskevopoulou P, Dervenis C, Kostopanagiotou G. Preincisional versus postincisional administration of parecoxib in colorectal surgery: effect on postoperative pain control and cytokine response. A randomized clinical trial. World J Surg 2010;34(10):2463-2469
19 Lassen K, Soop M, Nygren J, et al; Enhanced Recovery After Surgery (ERAS) Group. Consensus review of optimal perioperative care in colorectal surgery: Enhanced Recovery After Surgery (ERAS) Group recommendations. Arch Surg 2009;144(10): 961-969

20 Maund E, McDaid C, Rice S, Wright K, Jenkins B, Woolacott N. Paracetamol and selective and non-selective non-steroidal antiinflammatory drugs for the reduction in morphine-related sideeffects after major surgery: a systematic review. $\mathrm{Br} \mathrm{J}$ Anaesth 2011;106(03):292-297

21 Ong CKS, Seymour RA, Lirk P, Merry AF. Combining paracetamol (acetaminophen) with nonsteroidal antiinflammatory drugs: a qualitative systematic review of analgesic efficacy for acute postoperative pain. Anesth Analg 2010;110(04):1170-1179

22 Politi JR, Davis RL II, Matrka AK. Randomized prospective trial comparing the use of intravenous versus oral acetaminophen in total joint arthroplasty. J Arthroplasty 2017;32(04):1125-1127

23 Fenlon S, Collyer J, Giles J, et al. Oral vs intravenous paracetamol for lower third molar extractions under general anaesthesia: is oral administration inferior? Br J Anaesth 2013;110(03):432-437

24 Singla NK, Parulan C, Samson R, et al. Plasma and cerebrospinal fluid pharmacokinetic parameters after single-dose administration of intravenous, oral, or rectal acetaminophen. Pain Pract 2012;12(07):523-532

25 Kuo CP, Jao SW, Chen KM, et al. Comparison of the effects of thoracic epidural analgesia and i.v. infusion with lidocaine on cytokine response, postoperative pain and bowel function in patients undergoing colonic surgery. Br J Anaesth 2006;97(05): 640-646

26 Grady MV, Mascha E, Sessler DI, Kurz A. The effect of perioperative intravenous lidocaine and ketamine on recovery after abdominal hysterectomy. Anesth Analg 2012;115(05):1078-1084

27 Turan A, Karamanlioğlu B, Memiş D, Usar P, Pamukçu Z, Türe M. The analgesic effects of gabapentin after total abdominal hysterectomy. Anesth Analg 2004;98(05):1370-1373

28 Sen $\mathrm{H}$, Sizlan A, Yanarates $\mathrm{O}$, et al. A comparison of gabapentin and ketamine in acute and chronic pain after hysterectomy. Anesth Analg 2009;109(05):1645-1650

29 Cavalcante AN, Sprung J, Schroeder DR, Weingarten TN. Multimodal analgesic therapy with gabapentin and its association with postoperative respiratory depression. Anesth Analg 2017;125 (01):141-146

30 Chen J-Y, KoT-L, Wen Y-R, et al. Opioid-sparing effects of ketorolac and its correlation with the recovery of postoperative bowel function in colorectal surgery patients: a prospective randomized double-blinded study. Clin J Pain 2009;25(06):485-489

31 Gan TJ, Joshi GP, Zhao SZ, Hanna DB, Cheung RY, Chen C. Presurgical intravenous parecoxib sodium and follow-up oral valdecoxib for pain management after laparoscopic cholecystectomy surgery reduces opioid requirements and opioid-related adverse effects. Acta Anaesthesiol Scand 2004;48(09):1194-1207

32 Wattchow DA, De Fontgalland D, Bampton PA, Leach PL, McLaughlin $\mathrm{K}$, Costa M. Clinical trial: the impact of cyclooxygenase inhibitors on gastrointestinal recovery after major surgery - a randomized double blind controlled trial of celecoxib or diclofenac vs. placebo. Aliment Pharmacol Ther 2009;30(10):987-998

33 Gorissen KJ, Benning D, Berghmans T, et al. Risk of anastomotic leakage with non-steroidal anti-inflammatory drugs in colorectal surgery. Br J Surg 2012;99(05):721-727

34 Zakine J, Samarcq D, Lorne E, et al. Postoperative ketamine administration decreases morphine consumption in major abdominal surgery: a prospective, randomized, double-blind, controlled study. Anesth Analg 2008;106(06):1856-1861

35 Dale O, Somogyi AA, Li Y, Sullivan T, Shavit Y. Does intraoperative ketamine attenuate inflammatory reactivity following surgery? A systematic review and meta-analysis. Anesth Analg 2012;115 (04):934-943 
36 Remérand F, Le Tendre C, Baud A, et al. The early and delayed analgesic effects of ketamine after total hip arthroplasty: a prospective, randomized, controlled, double-blind study. Anesth Analg 2009;109(06):1963-1971

37 Koinig H, Wallner T, Marhofer P, Andel H, Hörauf K, Mayer N. Magnesium sulfate reduces intra- and postoperative analgesic requirements. Anesth Analg 1998;87(01):206-210

38 Waldron NH, Jones CA, Gan TJ, Allen TK, Habib AS. Impact of perioperative dexamethasone on postoperative analgesia and side-effects: systematic review and meta-analysis. $\mathrm{Br} \mathrm{J}$ Anaesth 2013;110(02):191-200

39 Bolac CS, Wallace AH, Broadwater G, Havrilesky LJ, Habib AS. The impact of postoperative nausea and vomiting prophylaxis with dexamethasone on postoperative wound complications in patients undergoing laparotomy for endometrial cancer. Anesth Analg 2013;116(05):1041-1047

40 Kurz A, Fleischmann E, Sessler DI, Buggy DJ, Apfel C, Akça O; Factorial Trial Investigators. Effects of supplemental oxygen and dexamethasone on surgical site infection: a factorial randomized trial. Br J Anaesth 2015;115(03):434-443

41 Tufanogullari B, White PF, Peixoto MP, et al. Dexmedetomidine infusion during laparoscopic bariatric surgery: the effect on recovery outcome variables. Anesth Analg 2008;106(06): $1741-1748$

42 Blaudszun G, Lysakowski C, Elia N, Tramèr MR. Effect of perioperative systemic $\alpha 2$ agonists on postoperative morphine consumption and pain intensity: systematic review and metaanalysis of randomized controlled trials. Anesthesiology 2012; 116(06):1312-1322

43 Collard V, Mistraletti G, Taqi A, et al. Intraoperative esmolol infusion in the absence of opioids spares postoperative fentanyl in patients undergoing ambulatory laparoscopic cholecystectomy. Anesth Analg 2007;105(05):1255-1262

44 Chia YY, Chan MH, Ko NH, Liu K. Role of $\beta$-blockade in anaesthesia and postoperative pain management after hysterectomy. $\mathrm{Br} \mathrm{J}$ Anaesth 2004;93(06):799-805

45 Block BM, Liu SS, Rowlingson AJ, Cowan AR, Cowan JA Jr, Wu CL. Efficacy of postoperative epidural analgesia: a meta-analysis. JAMA 2003;290(18):2455-2463

46 Marret E, Remy C, Bonnet F; Postoperative Pain Forum Group. Meta-analysis of epidural analgesia versus parenteral opioid analgesia after colorectal surgery. Br J Surg 2007;94(06): 665-673

47 Guay J, Nishimori M, Kopp SL. Epidural local anesthetics versus opioid-based analgesic regimens for postoperative gastrointestinal paralysis, vomiting, and pain after abdominal surgery: a Cochrane review. Anesth Analg 2016;123(06):1591-1602

48 Ahmed J, Lim M, Khan S, McNaught C, Macfie J. Predictors of length of stay in patients having elective colorectal surgery within an enhanced recovery protocol. Int J Surg 2010;8(08): 628-632

49 Khan SA, Khokhar HA, Nasr ARH, Carton E, El-Masry S. Effect of epidural analgesia on bowel function in laparoscopic colorectal surgery: a systematic review and meta-analysis. Surg Endosc 2013;27(07):2581-2591

50 Hübner M, Blanc C, Roulin D, Winiker M, Gander S, Demartines N. Randomized clinical trial on epidural versus patient-controlled analgesia for laparoscopic colorectal surgery within an enhanced recovery pathway. Ann Surg 2015;261(04):648-653

51 Halabi WJ, Kang CY, Nguyen VQ et al. Epidural analgesia in laparoscopic colorectal surgery: a nationwide analysis of use and outcomes. JAMA Surg 2014;149(02):130-136

52 Lee JH, Park JH, Kil HK, Choi SH, Noh SH, Koo BN. Efficacy of intrathecal morphine combined with intravenous analgesia versus thoracic epidural analgesia after gastrectomy. Yonsei Med J 2014;55(04):1106-1114

53 Kong SK, Onsiong SM, Chiu WK, Li MK. Use of intrathecal morphine for postoperative pain relief after elective laparoscopic colorectal surgery. Anaesthesia 2002;57(12):1168-1173

54 Wongyingsinn M, Baldini G, Stein B, Charlebois P, Liberman S, Carli F. Spinal analgesia for laparoscopic colonic resection using an enhanced recovery after surgery programme: better analgesia, but no benefits on postoperative recovery: a randomized controlled trial. Br J Anaesth 2012;108(05):850-856

55 Virlos I, Clements D, Beynon J, Ratnalikar V, Khot U. Short-term outcomes with intrathecal versus epidural analgesia in laparoscopic colorectal surgery. Br J Surg 2010;97(09):1401-1406

56 Charlton S, Cyna AM, Middleton P, Griffiths JD. Perioperative transversus abdominis plane (TAP) blocks for analgesia after abdominal surgery. Cochrane Database Syst Rev 2010;12(12): CD007705

57 Brogi E, Kazan R, Cyr S, Giunta F, Hemmerling TM. Transversus abdominal plane block for postoperative analgesia: a systematic review and meta-analysis of randomized-controlled trials. Can J Anaesth 2016;63(10):1184-1196

58 Walter CJ, Maxwell-Armstrong C, Pinkney TD, et al. A randomised controlled trial of the efficacy of ultrasound-guided transversus abdominis plane (TAP) block in laparoscopic colorectal surgery. Surg Endosc 2013;27(07):2366-2372

59 Rashid A, Gorissen KJ, Ris F, et al. No benefit of ultrasound guided transversus abdominis plane blocks over local anaesthetic wound infiltration in elective laparoscopic colonic surgery; results of a double blind randomized controlled trial. Colorect Dis 2016;19 (07):681-689

60 Oh TK, Yim J, Kim J, et al. Effects of preoperative ultrasoundguided transversus abdominis plane block on pain after laparoscopic surgery for colorectal cancer: a double-blind randomized controlled trial. Surg Endosc 2017;31(01):127-134

61 Pedrazzani C, Menestrina N, Moro M, et al. Local wound infiltration plus transversus abdominis plane (TAP) block versus local wound infiltration in laparoscopic colorectal surgery and ERAS program. Surg Endosc 2016;30(11):5117-5125

62 Gilron I, Orr E, Tu D, Mercer CD, Bond D. A randomized, doubleblind, controlled trial of perioperative administration of gabapentin, meloxicam and their combination for spontaneous and movement-evoked pain after ambulatory laparoscopic cholecystectomy. Anesth Analg 2009;108(02):623-630

63 Gilron I, Tu D, Dumerton-Shore D, et al. The effect of triple vs. double nonopioid therapy on postoperative pain and functional outcome after abdominal hysterectomy: a randomised doubleblind control trial. Eur J Anaesthesiol 2015;32(04):269-276

64 Oh ES, Li M, Fafowora TM, et al. Preoperative risk factors for postoperative delirium following hip fracture repair: a systematic review. Int J Geriatr Psychiatry 2015;30(09):900-910 